\title{
INTERPOLATION THEOREMS FOR A FAMILY \\ OF SPANNING SUBGRAPHS
}

\author{
Sanming Zhou, Hong Kong
}

(Received March 20, 1995)

\begin{abstract}
Let $G$ be a graph with order $p$, size $q$ and component number $\omega$. For each $i$ between $p-\omega$ and $q$, let $\mathscr{C}_{i}(G)$ be the family of spanning $i$-edge subgraphs of $G$ with exactly $\omega$ components. For an integer-valued graphical invariant $\varphi$, if $H \rightarrow H^{\prime}$ is an adjacent edge transformation (AET) implies $\left|\varphi(H)-\varphi\left(H^{\prime}\right)\right| \leqslant 1$, then $\varphi$ is said to be continuous with respect to AET. Similarly define the continuity of $\varphi$ with respect to simple edge transformation (SET). Let $M_{j}(\varphi)$ and $m_{j}(\varphi)$ be the invariants defined by $M_{j}(\varphi)(H)=$ $\max _{T \in \mathscr{C}_{j}(H)} \varphi(T), m_{j}(\varphi)(H)=\min _{T \in \mathscr{C}_{j}(H)} \varphi(T)$. It is proved that both $M_{p-\omega}(\varphi)$ and $m_{p-\omega}(\varphi)$ interpolate over $\mathscr{C}_{i}(G), p-\omega \leqslant i \leqslant q$, if $\varphi$ is continuous with respect to AET, and that $M_{j}(\varphi)$ and $m_{j}(\varphi)$ interpolate over $\mathscr{C}_{i}(G), p-\omega \leqslant j \leqslant i \leqslant q$, if $\varphi$ is continuous with respect to SET. In this way a lot of known interpolation results, including a theorem due to Schuster etc., are generalized.
\end{abstract}

\section{INTRODUCTION}

Let $\mathscr{F}$ be a family of graphs and $\varphi$ an integer-valued graphical invariant. We view $\varphi$ as a mapping from $\mathscr{F}$ to the set of integers. If the image set $\varphi(\mathscr{F})=\{\varphi(H)$ : $H \in \mathscr{F}\}$ is an integer interval, that is, if it consists of consecutive integers, then $\varphi$ is said to interpolate over $\mathscr{F}([5])$. In previous work it was shown that a number of invariants interpolate over some families of subgraphs, e.g. the family of spanning trees, of a connected graph. In this direction, the following result is well-known.

Theorem $1([2,7,9])$. For each integer $m \geqslant 0$, let $\varepsilon_{m}$ be the invariant defined by $\varepsilon_{m}(H)=\left|\left\{v \in V(H): d_{H}(v) \leqslant m\right\}\right|$ for any graph $H$. Then $\varepsilon_{m}$ interpolates over the family of spanning trees of any connected graph.

Note that $\varepsilon_{1}(H)$ is just the number of pendant vertices of $H$ if $H$ contains no isolated vertices. So Theorem 1 answers affirmatively a problem proposed by Chartrand [3]. As a generalization of this result, Barefoot [1] proved that $\varepsilon_{1}$ interpolates 
over the family of connected $i$-edge spanning subgraphs of any connected graph $G$ for each $i$ with $|V(G)|-1 \leqslant i \leqslant|E(G)|$ (a short proof for this result can be found in [11]). In this paper, we are going to generalize Theorem 1, as well as a lot of other interpolation results, along another direction.

Throughout the paper graphs are finite, undirected and with no loops and multiedges. We will always use $G$ to denote a graph with order $p$, size $q$ and component number $\omega$. For each $i, p-\omega \leqslant i \leqslant q$, let $\mathscr{C}_{i}(G)$ be the family of spanning subgraphs of $G$ with $i$ edges and $\omega$ components. In particular, $\mathscr{C}_{p-1}(G)$ is the family of spanning trees of $G$ if $G$ is connected. For two graphs $H$ and $H^{\prime}$ with the same vertex set, if $H^{\prime}=H-e+e^{\prime}$ for some $e \in E(H) \backslash E\left(H^{\prime}\right)$ and $e^{\prime} \in E\left(H^{\prime}\right) \backslash E(H)$, then call $H \rightarrow H^{\prime}$ a simple edge transformation (SET for short) or a simple edge exchange as used in [5]. If $H^{\prime}=H-e+e^{\prime}$ for adjacent $e$ and $e^{\prime}$, then call $H \rightarrow H^{\prime}$ an adjacent edge transformation (AET). An integer-valued invariant $\varphi$ is said to be continuous with respect to SET (AET, respectively) if $H \rightarrow H^{\prime}$ is SET (AET, respectively) implies that $\left|\varphi(H)-\varphi\left(H^{\prime}\right)\right| \leqslant 1$. For an invariant $\varphi$ and a graph $H$, define

$$
\begin{aligned}
& M_{j}(\varphi)(H)=\max _{T \in \mathscr{C}_{j}(H)} \varphi(T), \\
& m_{j}(\varphi)(H)=\min _{T \in \mathscr{C}_{j}(H)} \varphi(T),
\end{aligned}
$$

for each $j$ with $|V(H)|-\omega(H) \leqslant j \leqslant|E(H)|$, where $\omega(H)$ is the number of components of $H$ and $\mathscr{C}_{j}(H)$ is the family of spanning subgraphs of $H$ with $j$ edges and $\omega(H)$ components. Note that if $j=|E(H)|$, then $M_{j}(\varphi)(H)=m_{j}(\varphi)(H)=\varphi(H)$. One of the main results in this paper is the following

Theorem 2. Both $M_{j}(\varphi)$ and $m_{j}(\varphi)$ interpolate over $\mathscr{C}_{i}(G)$ provided that one of the following conditions is satisfied:

(a) $\varphi$ is continuous with respect to $S E T, p-\omega \leqslant j \leqslant i \leqslant q$;

(b) $\varphi$ is continuous with respect to $A E T, p-\omega=j \leqslant i \leqslant q$.

To prove this we need the following rather simple fact, which reveals the connection between global and local interpolations.

Proposition 1 [1]]. An invariant $\varphi$ interpolates over a family $\mathscr{F}$ if and only if there exists a connected graph $G(\mathscr{F})$ with vertex set $\mathscr{F}$ such that $\varphi$ interpolates over $N[H]$ for each $H \in \mathscr{F}$, where $N[H]$ is the subset of $\mathscr{F}$ consisting of $H$ and its neighbors in graph $G(\mathscr{F})$.

Theorem 2 is proved in the next section, and its further generalizations are given in Section 3. At the end of the paper some consequences of the main results are discussed. 


\section{Proof of Theorem 2}

Let $T_{i}(G)$ be the graph with vertex set $\mathscr{C}_{i}(G)$ in which $H$ and $H^{\prime}$ adjacent if and only if $H \rightarrow H^{\prime}$ is a SET. Similarly define the graph $A_{i}(G)$ on ' $\mathscr{C}_{i}(G)$ in which $H$ and $H^{\prime}$ adjacent if and only if $H \rightarrow H^{\prime}$ is an AET. Let $G_{1}, \ldots, G_{r}$ be the components of the graph obtained from $G$ by deleting all bridges and the resultant isolated vertices. Let $A_{q-1}^{k}(G)$ be the subgraph of $A_{q-1}(G)$ induced by $\mathscr{C}_{q-1}^{k}(G)=\left\{G-e: e \in E\left(G_{k}\right)\right\}$, $1 \leqslant k \leqslant r$. Then we have

Lemma 1. (a) For each $i, p-\omega \leqslant i \leqslant q-1, T_{i}(G)$ is connected. In fact, it is hamiltonian.

(b) $A_{q-1}^{k}(G), 1 \leqslant k \leqslant r$, are exactly the components of $A_{q-1}(G)$.

Proof. (a) Note that $T_{i}(G)$ is just the tree graph [6] of a matroid on $G$. In fact, this matroid is the elongation [10] of the cycle matroid of $G$ to height $i$. Since $i \leqslant q-1, T_{i}(G)$ contains cycles. By the hamiltonicity of the tree graphs of matroids [6], $T_{i}(G)$ is hamiltonian.

(b) If $G$ is 2-connected, then $A_{q-1}(G)$ is connected ([5], see also [12]). By using this fact in general case we know each $A_{q-1}^{k}(G)$ is connected. If $H \in \mathscr{C}_{q-1}^{k}(G)$, $H^{\prime} \in \mathscr{C}_{q-1}^{k^{\prime}}(G), k \neq k^{\prime}$, then $H \rightarrow H^{\prime}$ cannot be an AET and hence $H, H^{\prime}$ are not adjacent in $A_{q-1}(G)$. So (b) follows immediately.

The following lemma is a refinement of Lin's elegant proof [7] for Theorem 1.

Lemma 2. If $\varphi$ interpolates over $\mathscr{C}_{q-1}(G)$ for any graph $G$, then it interpolates over $\mathscr{C}_{i}(G)$ for each $i, p-\omega \leqslant i \leqslant q$, as well.

Pro of. The result is trivial when $i=q$. Suppose $p-\omega \leqslant i \leqslant q-1$, then $T_{i}(G)$ is connected by Lemma 1(a). For each $H \in \mathscr{C}_{i}(G)$, let $N[H]$ be the closed neighbor set of $H$ in $T_{i}(G)$, i.e. the set consisting of $H$ and the neighbors of it in $T_{i}(G)$. Then

$$
N[H]=\bigcup_{e \in E(G) \backslash E(H)} \mathscr{C}_{i}(H+e),
$$

and hence

$$
\varphi(N[H])=\bigcup_{e \in E(G) \backslash E(H)} \varphi\left(\mathscr{C}_{i}(H+e)\right) .
$$

Note that $H+e$ has $i+1$ edges, so $\varphi\left(\mathscr{C}_{i}(H+e)\right)$ is an integer interval by the hypothesis. Since all $\varphi\left(\mathscr{C}_{i}(H+e)\right)$, e $\in E(G) \backslash E(H)$, share a common element $\varphi(H), \varphi(N[H])$ is also an integer interval. From Proposition 1 we conclude that $\varphi$ interpolates over $\mathscr{C}_{i}(G)$. 
Lemma 3. Let $H, H^{\prime} \in \mathscr{C}_{q-1}(G)$ and $p-\omega \leqslant j \leqslant q-1$. Then we have

$$
\begin{aligned}
& \left|M_{j}(\varphi)(H)-M_{j}(\varphi)\left(H^{\prime}\right)\right| \leqslant 1 \\
& \left|m_{j}(\varphi)(H)-m_{j}(\varphi)\left(H^{\prime}\right)\right| \leqslant 1
\end{aligned}
$$

provided that one of the following conditions is satisfied:

(a) $\varphi$ is continuous with respect to SET and $H$ and $H^{\prime}$ are adjacent in $T_{q-1}(G)$;

(b) $\varphi$ is continuous with respect to $A E T$ and $H$ and $H^{\prime}$ are adjacent in $A_{q-1}(G)$.

Proof. As an example we prove the inequalities under condition (b). Since $M_{q-1}(\varphi)(H)=m_{q-1}(\varphi)(H)=\varphi(H), M_{q-1}(\varphi)\left(H^{\prime}\right)=m_{q-1}(\varphi)\left(H^{\prime}\right)=\varphi\left(H^{\prime}\right)$, both (1) and (2) are valid if $j=q-1$. In the following we assume $j \leqslant q-2$. Suppose $H^{\prime}=H-e+e^{\prime}$, where $e$ and $e^{\prime}$ are adjacent edges. Let

$$
\begin{aligned}
\mathscr{D}_{0} & =\mathscr{C}_{j}(H) \cap \mathscr{C}_{j}\left(H^{\prime}\right), \\
\mathscr{D} & =\mathscr{C}_{j}(H) \backslash \mathscr{C}_{j}\left(H^{\prime}\right), \\
\mathscr{D}^{\prime} & =\mathscr{C}_{j}\left(H^{\prime}\right) \backslash \mathscr{C}_{j}(H) .
\end{aligned}
$$

Since $G=H+e^{\prime}=H^{\prime}+e$, we have $\mathscr{D}_{0}=\left\{T \in \mathscr{C}_{j}(G): e, e^{\prime} \notin E(T)\right\}, \mathscr{D}=\{T \in$ $\left.\mathscr{C}_{j}(G): e \in E(T), e^{\prime} \notin E(T)\right\}$ and $\mathscr{D}^{\prime}=\left\{T \in \mathscr{C}_{j}(G): e \notin E(T), e^{\prime} \in E(T)\right\}$. It can be seen that the mapping $f: \mathscr{D} \rightarrow \mathscr{D}^{\prime}$ defined by $f(T)=T-e+e^{\prime}$ is a bijection from $\mathscr{D}$ to $\mathscr{D}^{\prime}$.

Let $n=M_{j}(\varphi)(H)$ and $T_{0} \in \mathscr{C}_{j}(H)$ be such that $\varphi\left(T_{0}\right)=n$. We distinguish four cases.

Case 1. $T_{0} \in \mathscr{D}_{0}, \max _{T \in \mathscr{D}} \varphi(T)<n$.

For each $T^{\prime} \in \mathscr{D}^{\prime}$, let $T=f^{-1}\left(T^{\prime}\right)=T^{\prime}-e^{\prime}+e$. Since $T \rightarrow T^{\prime}$ is an AET and $\varphi$ is continuous with respect to AET, we have $\varphi\left(T^{\prime}\right) \leqslant \varphi(T)+1 \leqslant(n-1)+1=n$. So $M_{j}(\varphi)\left(H^{\prime}\right)=n$.

Case 2. $T_{0} \in \mathscr{D}_{0}, \max _{T \in \mathscr{D}} \varphi(T)=n$.

By a similar argument as above we get $M_{j}(\varphi)\left(H^{\prime}\right)=n$ or $n+1$.

Case 3. $T_{0} \in \mathscr{D}, \max _{T \in \mathscr{D}_{0}} \varphi(T)<n$.

In such case $\varphi(T) \leqslant n-1$ for each $T \in \mathscr{D}_{0}$. Let $T_{0}^{\prime}=T_{0}-e+e^{\prime}$, then $\varphi\left(T_{0}^{\prime}\right)=n-1$, $n$ or $n+1$. For each $T^{\prime} \in \mathscr{D}^{\prime}$, let $T=T^{\prime}-e^{\prime}+e$. Then $\varphi\left(T^{\prime}\right) \leqslant \varphi(T)+1 \leqslant n+1$. So $M_{j}(\varphi)\left(H^{\prime}\right)=n-1, n$ or $n+1$.

Case 4. $T_{0} \in \mathscr{D}, \max _{T \in \mathscr{D}_{0}} \varphi(T)=n$.

Take $T_{1} \in \mathscr{D}_{0}$ with $\varphi\left(T_{1}\right)=n$. Then

$$
n \leqslant \max _{T \in \mathscr{D}} \varphi(T) \leqslant \max _{T \in \mathscr{C}_{j}(H)} \varphi(T)=M_{j}(\varphi)(H)=n .
$$

So $\max _{T \in \mathscr{D}} \varphi(T)=n$. Replacing $T_{0}$ by $T_{1}$ the case reduces to Case 2 . 
In each case above we have $\left|M_{j}(\varphi)(H)-M_{j}(\varphi)\left(H^{\prime}\right)\right| \leqslant 1$. In a similar way we can prove $\left|m_{j}(\varphi)(H)-m_{j}(\varphi)\left(H^{\prime}\right)\right| \leqslant 1$. This completes the proof of Lemma 3 .

Now we can proceed to the proof of Theorem 2.

Proof of Theorem 2. By Lemma 2 it suffices to prove that both $M_{j}(\varphi)$ and $m_{j}(\varphi)$ interpolate over $\mathscr{C}_{q-1}(G)$ under the given conditions.

(a) By Lemma 3 both $M_{j}(\varphi)$ and $m_{j}(\varphi)$ interpolate over the closed neighbor set of $H$ in $T_{q-1}(G)$. Since $T_{q-1}(G)$ is connected by Lemma 1(a), the result follows immediately from Proposition 1.

(b) Combining Lemma 1(b) and Lemma 3 with Proposition 1 we know both $M_{p-\omega}(\varphi)\left(\mathscr{C}_{q-1}^{k}(G)\right)$ and $m_{p-\omega}(\varphi)\left(\mathscr{C}_{q-1}^{k}(G)\right)$ are integer intervals, $1 \leqslant k \leqslant r$. Let

$T_{0} \in \mathscr{C}_{p-\omega}(G)$ be such that $\varphi\left(T_{0}\right)=\max _{T \in \mathscr{C}_{p-\omega}(G)} \varphi(T)$. Since $T_{0}$ is a forest and $G_{k}$ is 2-connected, there must exists an edge $e_{k} \in E\left(G_{k}\right)$ which is not in $T_{0}$. Thus $T_{0}$ is a subgraph of $G_{k}-e_{k} \in \mathscr{C}_{q-1}^{k}(G)$. So $M_{p-\omega}(\varphi)\left(G_{k}-e_{k}\right)=\varphi\left(T_{0}\right)$. Thus all integer intervals $M_{p-\omega}(\varphi)\left(\mathscr{C}_{q-1}^{k}(G)\right), 1 \leqslant k \leqslant r$, share a common element $\varphi\left(T_{0}\right)$, implying that $M_{p-\omega}(\varphi)\left(\mathscr{C}_{q-1}(G)\right)=\bigcup_{1 \leqslant k \leqslant r} M_{p-\omega}(\varphi)\left(\mathscr{C}_{q-1}^{k}(G)\right)$ is an integer interval. Similarly $m_{p-\omega}(\varphi)\left(\mathscr{C}_{q-1}(G)\right)$ is an integer interval. This completes the proof.

\section{FURTHER RESULtS}

The proof of Lemma 3 conveys more information than what has been used. In this section we will give a general result, which implies Theorem 2(a), by using Lemma 3 repeatedly.

For integers $l, j, p-\omega \leqslant l \leqslant j \leqslant q-1$, and $H \in \mathscr{C}_{q-1}(G), T \in \mathscr{C}_{j}(H)$, let

$$
\Psi_{l}(T)=M_{l}(\varphi)(T), \psi_{l}(T)=m_{l}(\varphi)(T)
$$

Let

$$
M m_{j, l}(\varphi)(H)=M_{j}\left(\psi_{l}\right)(H)=\max _{T \in \mathscr{C}_{j}(H)} \min _{S \in \mathscr{C}_{l}(T)} \varphi(S)
$$

and

$$
m M_{j, l}(\varphi)(H)=m_{j}\left(\Psi_{l}\right)(H)=\min _{T \in \mathscr{C}_{j}(H)} \max _{S \in \mathscr{C}_{l}(T)} \varphi(S) .
$$

Then we have

Lemma 4. Let $H, H^{\prime} \in \mathscr{C}_{q-1}(G), p-\omega \leqslant l \leqslant j \leqslant q-1$. If $H \rightarrow H^{\prime}$ is a SET and $\varphi$ is continuous with respect to SET, then

$$
\begin{aligned}
\left|M m_{j, l}(\varphi)(H)-M m_{j, l}(\varphi)\left(H^{\prime}\right)\right| & \leqslant 1, \\
\left|m M_{j, l}(\varphi)(H)-m M_{j, l}(\varphi)\left(H^{\prime}\right)\right| & \leqslant 1 .
\end{aligned}
$$


Proof. If $j=q-1$, then $M m_{j, l}(\varphi)(H)=m_{l}(\varphi)(H), m M_{j, l}(\varphi)(H)=$ $M_{l}(\varphi)(H)$. The result follows from Lemma 3. In the following we suppose $j \leqslant q-2$. We first prove that if $T \in \mathscr{C}_{j}(H), T^{\prime} \in \mathscr{C}_{j}\left(H^{\prime}\right)$ and $T \rightarrow T^{\prime}$ is a SET, then

$$
\begin{gathered}
\left|\Psi_{l}(T)-\Psi_{l}\left(T^{\prime}\right)\right| \leqslant 1 \\
\left|\psi_{l}(T)-\psi_{l}\left(T^{\prime}\right)\right| \leqslant 1 .
\end{gathered}
$$

In fact in such case $T \cup T^{\prime}$ (the union of $T$ and $T^{\prime}$ ) has exactly $j+1$ edges. Since $T, T^{\prime} \in$ $\mathscr{C}_{j}\left(T \cup T^{\prime}\right)$ and $\varphi$ is continuous with respect to SET, we obtain (5-6) immediately from Lemma 3. Replacing $\varphi$ by $\psi_{l}$ in the proof of Lemma 3 and applying inequality (6) we get

$$
\left|M_{j}\left(\psi_{l}\right)(H)-M_{j}\left(\psi_{l}\right)\left(H^{\prime}\right)\right| \leqslant 1,
$$

which is just (3). Analogously we can prove (4).

Combining Proposition 1 and Lemmas 1, 2, 4 we get

Theorem 3. Suppose $\varphi$ is continuous with respect to SET and $p-\omega \leqslant l \leqslant j \leqslant$ $i \leqslant q$, then both $M m_{j, l}(\varphi)$ and $m M_{j, l}(\varphi)$ interpolate over $\mathscr{C}_{i}(G)$.

Note. We need not consider $M M_{j, l}(\varphi)$ and $m m_{j, l}(\varphi)$ since

$$
\begin{aligned}
M M_{j, l}(\varphi)(H) & =\max _{T \in \mathscr{C}_{j}(H)} \max _{S \in \mathscr{C}_{l}(T)} \varphi(S)=M_{l}(\varphi)(H), \\
m m_{j, l}(\varphi)(H) & =\min _{T \in \mathscr{C}_{j}(H)} \min _{S \in \mathscr{C}_{l}(T)} \varphi(S)=m_{l}(\varphi)(H) .
\end{aligned}
$$

We can go even further in the theme of Theorem 3. For $p-\omega \leqslant j_{1} \leqslant \ldots \leqslant$ $j_{2 k} \leqslant j_{2 k+1} \leqslant q$, put $M^{1} m_{j_{2}, j_{1}}^{1}(\varphi)=M m_{j_{2}, j_{1}}(\varphi)$ and $m^{1} M_{j_{2}, j_{1}}^{1}(\varphi)=m M_{j_{2}, j_{1}}(\varphi)$. Inductively define

$$
M^{k} m_{j_{2 k}, \ldots, j_{1}}^{k}(\varphi)=M m_{j_{2 k}, j_{2 k-1}}\left(M^{k-1} m_{j_{2 k-2}, \ldots, j_{1}}^{k-1}(\varphi)\right)
$$

and

$$
m^{k} M_{j_{2 k}, \ldots, j_{1}}^{k}(\varphi)=m M_{j_{2 k}, j_{2 k-1}}\left(m^{k-1} M_{j_{2 k-2}, \ldots, j_{1}}^{k-1}(\varphi)\right)
$$

Define

$$
M^{k+1} m_{j_{2 k+1}, \ldots, j_{1}}^{k}(\varphi)=M_{j_{2 k+1}}\left(m^{k} M_{j_{2 k}, \ldots, j_{1}}^{k}(\varphi)\right)
$$

and

$$
m^{k+1} M_{j_{2 k+1}, \ldots, j_{1}}^{k}(\varphi)=m_{j_{2 k+1}}\left(M^{k} m_{j_{2 k}, \ldots, j_{1}}^{k}(\varphi)\right)
$$

By induction we can prove a lemma which is similar to Lemma 4 for the invariants above. This leads to the following 
Theorem 4. If $\varphi$ is continuous with respect to SET and $p-\omega \leqslant j_{1} \leqslant \ldots \leqslant$ $j_{2 k} \leqslant j_{2 k+1} \leqslant i \leqslant q$, then $M^{k} m_{j_{2 k}, \ldots, j_{1}}^{k}(\varphi), m^{k} M_{j_{2 k}, \ldots, j_{1}}^{k}(\varphi), M^{k+1} m_{j_{2 k+1}, \ldots, j_{1}}^{k}(\varphi)$ and $m^{k+1} M_{j_{2 k+1}, \ldots, j_{1}}^{k}(\varphi)$ all interpolate over $\mathscr{C}_{i}(G)$.

If $j_{1}=\ldots=j_{2 k}=l \leqslant j=j_{2 k+1}$, then

$$
\begin{aligned}
M^{k} m_{j_{2 k}, \ldots, j_{1}}^{k}(\varphi) & =M_{l}(\varphi), \\
m^{k} M_{j_{2 k}, \ldots, j_{1}}^{k}(\varphi) & =m_{l}(\varphi), \\
M^{k+1} m_{j_{2 k+1}, \ldots, j_{1}}^{k}(\varphi) & =M m_{j, l}(\varphi)
\end{aligned}
$$

and

$$
m^{k+1} M_{j_{2 k+1}, \ldots, j_{3}}^{k}(\varphi)=m M_{j, l}(\varphi)
$$

So Theorem 4 generalizes both Theorem 2(a) and Theorem 3.

\section{Consequences}

Theorem 2 generalizes a number of known interpolation results. For example, if $j=i=p-\omega$ and $\varphi$ is continuous with respect to $\mathrm{AET}$, then $M_{j}(\varphi)=m_{j}(\varphi)=\varphi$ and Theorem 2 says that $\varphi$ interpolates over $\mathscr{C}_{p-\omega}(G)$. This implies Theorem 1 since $\varepsilon_{m}$ is continuous with respect to AET. For an integer $m \geqslant 0$, let $\delta_{m}(H)=$ $\left|\left\{v \in V(H): d_{H}(v) \geqslant m\right\}\right|$. For a spanning subgraph $H$ of $G$, let $\bar{\varepsilon}_{m}(H)=\varepsilon_{m}(\bar{H})$ and $\bar{\delta}_{m}(H)=\delta_{m}(\bar{H})$, where $\bar{H}$ is the complement graph of $H$ with respect to $G$. In general for any graph $K$ with $G$ as its spanning subgraph and any spanning subgraph $H$ of $G$, let $\bar{H}(K)=K-E(H)$ be the complement graph of $H$ with respect to $K$. Define $\bar{\varepsilon}_{m}^{K}(H)=\varepsilon_{m}(\bar{H}(K))$ and $\bar{\delta}_{m}^{K}(H)=\delta_{m}(\bar{H}(K))$ as in [13]. Then $\bar{\varepsilon}_{m}^{G}=\bar{\varepsilon}_{m}$ and $\bar{\delta}_{m}^{G}=\bar{\delta}_{m}$. It is not difficult to prove that all these invariants are continuous with respect to AET. So Theorem 2 implies the following

Corollary 1. If $\varphi=\varepsilon_{m}, \delta_{m}, \bar{\varepsilon}_{m}, \bar{\delta}_{m}, \bar{\varepsilon}_{m}^{K}$ or $\bar{\delta}_{m}^{K}$, then both $M_{p-\omega}(\varphi)$ and $m_{p-\omega}(\varphi)$ interpolate over $\mathscr{C}_{i}(G), p-\omega \leqslant i \leqslant q$. In particular, $\varepsilon_{m}, \delta_{m}, \bar{\varepsilon}_{m}, \bar{\delta}_{m}, \bar{\varepsilon}_{m}^{K}$ and $\bar{\delta}_{m}^{K}$ all interpolate over $\mathscr{C}_{p-\omega}(G)$, the family of spanning forests having the same number of components as $G$.

As mentioned earlier, this corollary implies the result in Theorem 1. It also implies the main results of [13], which in turn imply the main result of [8] since for $H \in \mathscr{C}_{i}(G)$, $\bar{\varepsilon}_{0}(H)=\left|\left\{v \in V(H): d_{H}(v)=d_{G}(v)\right\}\right|$ is exactly the number of degree-preserving vertices of $H[8]$.

A large number of invariants have been shown to be continuous with respect to SET. Hence they all interpolate over $\mathscr{C}_{i}(G)$ according to Proposition 1 and Lemma 1(a). These invariants include the connectivity, edge connectivity, independence 
number, edge independence number, vertex covering number, edge covering number, chromatic number, edge chromatic number, domination number, and so on. The reader is refered to $[5,11,14]$ for such invariants. Here we give only two examples to show how to generalize the existing interpolation results for these invariants by our main results in previous sections. It was shown [14] that the conditional chromatic number $\chi_{P}$ and the conditional edge chromatic number $\chi_{P}^{\prime}$ are continuous with respect to SET for any hereditary graphical property $P$ (a property $P$ is hereditary if whenever a graph possesses $P$ then all subgraphs of it have $P$ as well). Here $\chi_{P}(H)$ is defined [4] to be the minimum order $n$ of a partition $\left\{V_{1}, \ldots, V_{n}\right\}$ of $V(H)$ such that each induced subgraph $H\left[V_{i}\right]$ possesses the property $P$. The conditional edge chromatic number $\chi_{P}^{\prime}(H)$ is defined similarly. From Proposition 1 and Lemma 1 , both $\chi_{P}$ and $\chi_{P}^{\prime}$ interpolate over $\mathscr{C}_{i}(G)$ (this was proved in [14] for the case when $G$ is connected, interpolation for $\chi_{P}$ and $\chi_{P}^{\prime}$ with respect to other families of subgraphs can also be found in [14]). From Theorems 2 and 4 this can be strengthened as follows: $M_{j_{1}}\left(\chi_{P}\right), m_{j_{1}}\left(\chi_{P}\right), M_{j_{1}}\left(\chi_{P}^{\prime}\right), m_{j_{1}}\left(\chi_{P}^{\prime}\right), M m_{j_{2}, j_{1}}\left(\chi_{P}\right), m M_{j_{2}, j_{1}}\left(\chi_{P}\right), M m_{j_{2}, j_{1}}\left(\chi_{P}^{\prime}\right)$, $m M_{j_{2}, j_{1}}\left(\chi_{P}^{\prime}\right), \ldots$, all interpolate over $\mathscr{C}_{i}(G), p-\omega \leqslant j_{1} \leqslant j_{2} \leqslant \ldots \leqslant i \leqslant q$. For dozens of other invariants which are continuous with respect to SET we can get the similar results. Due to the limited space we cannot go into details.

\section{References}

[1] C.A. Barefoot: Interpolation theorem for the number of pendant vertices of connected spanning subgraphs of equal size. Discrete Math. 49 (1984), 109-112.

[2] M.C. Cai: A solution of Chartrand's problem on spanning trees. Acta Mathematica Applicata Sinica 1 (1994), no. 2, 97-98.

[3] The Theory and Applications of Graphs. Proc. Fourth Intern. Conf. on Graph Theory Applications, 1980 (G. Chartrand, etc., eds.). Wiley, New York, 1981, pp. 610.

[4] F. Harary: Conditional colorability in graphs. Graphs and Applications (F. Harary and J. S. Maybee, eds.). Wiley, New York, 1985, pp. 127-136.

[5] F. Harary and M. Plantholt: Classification of interpolation theorems for spanning trees and other families of spanning subgraphs. J. Graph Theory 13 (1989), 703-712.

[6] C.A. Holzmann and F. Harary: On the tree graph of a matroid. SIAM J. Appl. Math. 22 (1972), 187-193.

[7] Y. X. Lin: A simpler proof of interpolation theorem for spanning trees. Kexue Tongbao (English edition) 30 (1985), 134.

[8] M. Lewinter: Interpolation theorem for the number of degree-preserving vertices of spanning trees. IEEE Trans. Circuit and Systems 34 (1987), 205.

[9] S. Schuster: Interpolation theorem for the number of end-vertices of spanning trees. J. Graph Theory 7 (1983), 203-208.

[10] D.J.A. Welsh: Matroid Theory. Academic Press, London, 1976.

[11] S.M. Zhou: Matroid tree graphs and interpolation theorems. Discrete Math. 137 (1995), 395-397.

[12] S.M. Zhou: An interpolation theorem of graphs. A Friendly Collection of Math. Papers I. Jilin University Press, 1990, pp. 154-156.

[13] S.M. Zhou: Several interolation theorems for graphs. Graph Theory Notes of New York XXIX (1995), 18-20. 
[14] S.M. Zhou: Conditional invariants and interpolation theorems for graphs. Submitted.

Author's address: Department of Mathematics, The University of Hong Kong, Hong Kong; Current address: Department of Mathematics, The University of Western Australia, Nedlands, Perth, WA 6907, Australia. 